\title{
A Road Map for the Identification of the Diffuse Interstellar Band Carriers
}

\author{
N. L. J. Cox and J. Cami
}

\begin{abstract}
We present a road map of several research avenues leading to the identification of the diffuse interstellar band carriers. The proposed programs represent some consensus among the DIB community, and will certainly take many years to complete. However, the scientific payoff will be huge, and will ultimately lead to the solution of the DIB problem.
\end{abstract}

Keywords. ISM: abundances, ISM: atoms, ISM: lines and bands, ISM: molecules

\section{Introduction}

The problem associated with the identification of the diffuse interstellar band carriers has been around for nearly a century and is well known, but despite numerous efforts and various claims, none of the DIBs has been unambiguously linked to a specific carrier. There is a general consensus that a definite identification will ultimately require a match with a (cold) laboratory gas phase spectrum where all bands for that specific carrier match in wavelengths and relative intensities. The number of possible carriers however is so dauntingly large, that one of the main goals in DIB research is to simply eliminate as many candidates as possible - a task which requires input from various angles.

As is illustrated by the contributions in this volume, tremendous progress has been made in the last fifteen years in observational and theoretical astronomical studies, in laboratory astrophysics and spectroscopy, and in our understanding of molecular physics and interstellar chemistry. This interdisciplinary collaboration is crucial to ultimately identify the DIB carriers, and IAU Symposium 297 brought together many experts in these areas. It was recognized that the field would benefit from a common plan that defines priorities and recommendations for future research, and that is supported by the entire DIB community. Here, we present such a road map that we developed from the many points raised during several dedicated sessions. The road map includes organizational issues ( $(2)$, observational studies ( $(3)$, laboratory work ( $\$ 4)$ and theory $(\S 5)$.

\section{Organization \& Data Access}

The interdisciplinary nature of the DIB field introduces some challenges, and it would be good if the DIB community would be more formally organized to better address and resolve these issues. There are several recommendations to achieve this. First, we should establish a DIB working group (under the IAU or the AAS) with representatives from each of the disciplines. There should also be more regular meetings related to the DIB problem, and a newsletter to ensure that we are well aware of each other's research results on a timely basis. Another modern way to communicate and share information is through a collaborative web site; this site could contain organizational information, but also be a repository for various data (e.g. fully reduced astronomical observations or laboratory spectra; of course all with the proper caveats) and relevant information (e.g. literature).

The different disciplines should also engage in advocacy for each other. For instance, while astronomers understand the need for laboratory work in the DIB context, they 
could be be more active and outspoken in their efforts to unequivocally support and drive laboratory astrophysics programs. This is especially the case when seeking funding and creating collaborative networks. Setting up such a DIB research (and training) network could foster further interdisciplinary collaborations advancing the field.

One major consequence of the interdisciplinary nature of the field is the challenge of "language confusion". It is of utmost importance that we clearly "define the language" in an unambiguous way, and set standards and benchmarks for measurements and methods that are accessible to all researchers in the various disciplines. Similarly, limitations in each field are not always appreciated by those working in other fields, and it would be good to spell out these limitations for the DIB community.

\section{Observational Studies}

At the heart of the DIB puzzle are astronomical observations. Much progress towards DIB identification can be expected from observational programs that are focused on exposing how precisely the DIB carriers are connected to the inventory of interstellar matter, and how their properties depend on the detailed physical conditions in the environment where they reside.

A vital piece of information that is thus far missing from our data sets in that context are studies that relate the DIB properties to elemental depletion in the ISM and to variations in e.g. the deuterium abundance. Such studies could reveal much about the chemical makeup of some of the carriers. Similarly, detailed studies are needed to elucidate the role of dust grains in the formation, survival and destruction of DIB carriers. A more detailed knowledge of how individual DIBs compare to the various components of interstellar extinction and the molecular content of the sight lines would be welcomed. For many of these studies, lines of sight in Local Group galaxies with different metallicities should be included as well. Finally, we should also do more efforts to treat the DIB problem in relation to other unidentified spectral phenomena such as the the UV extinction bump, the extended red emission, blue luminescence, the red rectangle bands, the mid-infrared PAH emission bands and various far-infrared/sub-mm absorption lines.

Similarly, we need a much better understanding of how the physical conditions influence the DIBs, and also in this area only the surface has been skimmed. There is thus a clear need for more research on the properties of DIBs in lines of sight where conditions are either well known and understood, or peculiar (maybe even extreme), or where some parameters can be eliminated as influencing the DIBs. More specifically, this asks for observational studies on single cloud lines of sight, but also lines of sight that have DIBs but no (or very low) reddening; lines of sight with extreme conditions (AGNs; circumstellar environments; strong ionizing conditions, ... ). Also here, Local Group galaxies provide unique laboratories covering specific areas in the parameter space. One important related question is the formation and presence of DIB carriers in the distant/early Universe. This is particularly pertinent in view of the possible complex (organic) molecular nature of the carriers.

Of particular interest are those remarkable objects or sight lines such as the Red Rectangle and Herschel 36 that stand out in one or more observed properties, while at the same time some of the relevant parameters for the physical conditions can be reliably determined. These could be the Rosetta stone for deciphering all of the unexplained phenomena - including the DIBs. It is of utmost importance to study these objects in as many different ways as possible.

A key aim for future observational programs is to "beat a few well-chosen sight lines to death" by obtaining a sensitive and detailed multi-wavelength view from the UV (or even 
$\mathrm{X}$-ray) regime to $\mathrm{mm}$ wavelengths, and connecting the information gained from each of those areas to draw a complete picture of the interstellar matter content, while offering all the tools to obtain a deep understanding of the physical conditions in the line of sight. In turn, this can then be used to study how the DIB properties change in relation to these. However, scanning the same diffuse clouds across the entire wavelength range is a huge technical challenge, and will undoubtedly require current and new facilities such as HST (UV), JWST (near-IR) and ALMA (sub-mm); there was furthermore a strong call for a new and dedicated space mission for UV spectroscopy. Such studies furthermore require a good choice of targets. The extraordinary targets mentioned above could be good choices; but ultimately, this exercise needs to be completed for "normal" interstellar sight lines.

The DIB community should also take advantage of the opportunities offered by current and planned instruments, missions and data archives to carry out large survey programs - spectral surveys ("all DIBs, few targets") as well as sight line surveys ("few DIBs, many targets"). The first kind can be used to find "genetic" relationships between DIBs and other interstellar components. While correlation studies and DIB family classifications and groupings (e.g. the $\mathrm{C}_{2}$-DIBs) have been done before, they have been limited to the strongest DIBs and have traditionally only included mutual correlations and linear regressions. DIB researchers could apply more state-of-the-art multivariate statistical methods and computational techniques (such as principle component analyses and blind signal decompositions) to the entire interstellar spectrum (not only including DIBs) to define which parameters are of importance for the DIB problem and which ones are not. Such studies will set the future reference standards for DIB research, and will allow the community to focus on the most important issues first. The sight line surveys on the other hand can be used to characterize and classify environments (e.g. $\sigma$ and $\zeta$ type clouds), and will undoubtedly turn up more intriguing objects (as outliers one way or another) that can then be subject of further detailed studies. Here, the advent of large all-sky spectroscopic surveys may greatly benefit DIB research. Although these surveys focus generally only on a small wavelength range and have often only moderate spectral resolution and/or poor sensitivity, these surveys could prove valuable for extracting unusual targets for follow-up research. Large surveys (such as the upcoming GAIA mission) also offer a change to create 2D/3D distribution maps of different DIBs. Detailed analyses of their respective spatial distribution may give new insights into fundamental carrier properties.

Finally, by using these surveys, the DIB community should develop diagnostic tools that will allow astronomers to use the DIBs to infer some characteristics of the objects in which they are found. This is all intimately related to the other DIB research, since this requires very clear and tight relations between the DIBs and other parameters. One such example is the tight correlation between the equivalent width of the $\lambda 5780 \mathrm{DIB}$ and the column density of neutral hydrogen. These parameters need to be defined and properly named, and the precise quantitative relations (or lack thereof) between the DIBs and these parameters need to be well described, and their uncertainties be made specific. The DIB community should be well aware of these limitations as well, and should speak up when other researchers improperly use the DIBs, for example when using a single DIB equivalent width to determine E(B-V). Developing such tools will additionally help to raise awareness and support for future DIB studies.

\section{Laboratory Astrophysics \& Astrochemistry}

Laboratory spectroscopy at high spectral resolution will clearly be indispensable for obtaining a definitive identification of the DIB carriers. Priority should be given to 
obtaining the relevant (cold, gas-phase) spectra of specific molecules that have been proposed for specific DIBs (such as e.g. $\mathrm{C}_{60}^{+}$), and undoubtedly overcoming the difficulties involved in obtaining such spectra will be of importance for other fields as well. Spectroscopists should also ensure that their work reflects some of the recent expansion of the DIB inventory well into the near-infrared (currently up to $1.78 \mu \mathrm{m}$, but more surely to come). More research into the possibilities and value of helium nanodroplet experiments in the DIB context would also be welcome.

In addition to pure spectroscopy, there are many more ways in which laboratory research will be defining future DIB research. Similar to what has been done in the past in ice analogue studies, or perhaps even the Urey-Miller origin of life experiment, it would be useful simulate interstellar conditions, seed the experimental chamber with initial components and then analyze the products to assess which ones could be viable DIB carrier candidates. The creation of a hydrocarbon plasma revealing a broad absorption band coinciding with a known DIB represents an important step in this direction. In a broader context, there is a need for a large array of experimental dust-molecule interaction studies (e.g. formation, fragmentation, (de-)hydrogenation, ... ) to reveal which can be the truly stable and abundant interstellar species.

One specific recommendation for the laboratory community is to keep searching for opportunities to apply the experimental work to other fields, and to industrial settings if at all possible.

\section{Theoretical Work}

This symposium highlighted the impressive progress that has been made in theoretical and computational methods. Computational chemistry is crucial to guide, focus and drive laboratory work as well as astronomical observations. Often, such calculations represent a trade-off between accuracy and computational cost. A key role for this type of theoretical work is to use "easy and cheap" methods that can identify which specific classes of carriers can give rise to narrow and strong optical transitions. Such a generic approach can cover a much larger sample of possible carriers than the highly accurate calculations that are currently possible, and represents a cheap way to narrow down carrier candidates when properly analyzed. The state-of-the-art calculations on the other hand are crucial to properly evaluate a limited pool of probable carriers.

Theoretical work in the context of the DIBs entails much more though than quantum chemistry. As for the laboratory work, there is a clear need to study from the theoretical and computational point of view which are the most likely carriers. This will require developing a large reaction network with countless species in various states (charge states, but also hydrogenation states for instance), but also a good understanding of what the relevant input parameters are for a given line of sight. Clearly, this can only be obtained from detailed modeling efforts to describe the physical conditions and chemical composition of specific well-studied lines of sight.

\section{Acknowledgements}

This road map is the synthesis of several hours of active discussion sessions that were held at IAU Symposium 297. We would like to thank the discussion chairs (Wim Ubachs, Jonathan Tennyson, Peter Sarre) for their preparation and guidance of these discussions, and all conference participants for their valuable input. Let the DIB future be bright and exciting! 